\title{
Trabeculectomy with fistula formation in the African
}

\author{
N. H. WELSH
}

Department of Ophthalmology, University of Natal and King Edward VIII Hospital, Durban, $\overrightarrow{0}$ South Africa

Operations on the trabecular meshwork in order to reduce raised intraocular pressure are $\mathrm{\omega}^{\mathrm{N}}$

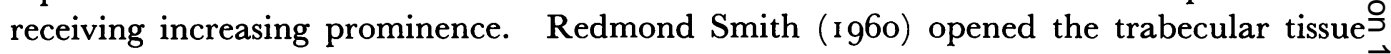
by passing a nylon thread along the canal of Schlemm via an ab externo incision, and by ac further incision and by picking up the distal end of the thread, he ruptured the trabecular? meshwork by pulling on the thread like a bow. Allen and Burian (1962) passed an instrument into the canal of Schlemm via an ab externo incision and ruptured the $\operatorname{trabecular} \overrightarrow{0}$ tissue. Strachan (1967) and Harms and Dannheim (1969) have modified this further N until now the operation of trabeculotomy "ab externo" has been well documented." Krasnov ( 1968 ) described the operation of sinusotomy or externalization of the canal of Schlemm in cases in which the obstruction to outflow may be intrascleral, and Walker andō Kanagasundaram ( 1964 ) have also followed this principle. Trabeculectomy or removal $\frac{\varnothing}{\mathbb{D}}$ of trabecular meshwork through an ab externo incision (Cairns, 1968) allows the aqueous to $\underset{\Rightarrow}{\Rightarrow}$ flow through the now exposed cut ends of Schlemm's canal and then to leave the eye via the normal exit channels. In African patients, in whom the problems of failure of drainage operations have been shown to be due mainly to fibrosis derived from the sclera우․ and from Tenon's capsule (Welsh, 1970), it was thought that trabecular operations would응. be theoretically advantageous. Trabeculectomy was chosen as the operation of choice ${ }^{\circ}$ because of the results obtained by others up to this time, and also because histological examination of the trabecular tissue could then be performed and so afford direct evidence $\frac{\delta}{3}$ of any obstructive pathology. Trabeculectomy as described by Cairns ( 1968 ) was therefore performed in a series of cases. It was found that trabeculectomy in itself reduced the intraocular pressure only partially. A controlled series of cases in which the trabeculec-吾 tomy was combined with intentional fistula formation was also carried out, and theseñ cases showed a much higher success rate than those in which trabeculectomy was used ${ }_{N}^{\circ}$ alone. This combined procedure seems also to be more successful than the standard N్ drainage procedures, and the purpose of this paper is to discuss these findings and to suggest that in the more heavily pigmented races the combined procedure has considerable merit.

\section{Material and methods}

A prospective study was made during the period October 24, 1969, to February I, 1971, of $128 \stackrel{\frac{7}{\circ}}{\circ}$ operations on 96 patients with chronic open-angle glaucoma. All the operations were performed $\frac{?}{\mathbb{Q}}$ by one surgeon (N.H.W.) and the operations were divided into two groups (Table I). Group I $\cong$ comprised the operation of trabeculectomy and, although 66 trabeculectomies (47 patients) were performed, only $4^{2}$ (33 patients) had sufficient follow up. Group II comprised the operation of 
trabeculectomy combined with intentional fistula formation (called filtration trabeculectomy); there were 62 operations (49 patients) but only 35 ( 26 patients) had sufficient follow up.

Table I Number of patients and operations over I 8-month period

\begin{tabular}{|c|c|c|c|c|c|c|}
\hline \multirow{2}{*}{ Group } & \multicolumn{3}{|l|}{ Patients } & \multicolumn{3}{|c|}{ Operations } \\
\hline & African & Indian & Total & African & Indian & Total \\
\hline I Trabeculectomies & 28 & 5 & 33 & 36 & 6 & $4^{2}$ \\
\hline $\begin{array}{l}\text { II Combined trabeculectomy } \\
\text { with fistula formation } \\
\text { (filtration trabeculectomy) }\end{array}$ & 22 & 4 & 26 & 29 & 6 & 35 \\
\hline
\end{tabular}

The operations were performed on African (Zulu) and Indian patients, but the latter were too few to be significant. The cases were not selected and the two groups were comparable in terms of age, sex, and degree and extent of glaucoma. All intraocular pressures varied from 30 to $60 \mathrm{~mm} . \mathrm{Hg}$, and in the majority of cases the visual acuity was limited to $6 / 60$ or counting fingers.

The technique of trabeculectomy was based on the description of Cairns (1968) with modifications. A fornix-based scleral hinge was cut, care being taken to make the superficial scleral flap one-third the thickness of the sclera or less. The flap was usually $5 \mathrm{~mm}$. wide and $6 \mathrm{~mm}$. long. When the superficial flap had been dissected, the transition zone between white sclera and greyish-black limbus was well seen. Similarly, as the deep sclero-limbal area under the flap containing Schlemm's canal and trabecular meshwork was removed, the trabecular area on the internal surface was evident. The edges of the scleral section had to be clean-cut and not ragged so that the open ends of Schlemm's canal remained patent. The deep scleral tissue containing trabecular tissue and Schlemm's canal was removed by starting at Schwalbe's line and dissecting towards the scleral spur. The dissection was stopped at the ciliary body and a peripheral iridectomy was performed. When the superficial scleral flap was sutured into position, the side-to-side stitches had to be superficial to avoid Schlemm's canal and its efferent channels.

Ringer's solution was injected through the sides of the incision to re-form the anterior chamber and to avoid peripheral anterior synechia formation. The operation can be performed inferiorly if required and it can also be repeated, but there is the obvious danger that one may reach the stage of excising too great an amount of Schlemm's canal with consequent loss of drainage area. Postoperatively, the success of the operation can be assessed by the usual methods, but gonioscopy is particularly interesting because one can see the gap on the internal surface of the trabecular area extending from Schwalbe's line to the root of the iris. On occasions, one can see blood flowing back into the anterior chamber from the free cut ends of Schlemm's canal. Gonioscopy thus confirms the correctness of one's technique and enables one to follow the fate of the internal raw surface. In the combined procedure of filtration trabeculectomy, a similar procedure was followed but, instead of stitching the superficial scleral flap into place, $2 \mathrm{~mm}$. of the distal free end of the flap was excised as in a sclerectomy operation and no stitches were inserted. In eight operations, iris was intentionally included in the wound. In order to obtain a good fistula and to avoid adhesions between sclera, external opening, and Tenon's capsule, particular care was taken to excise Tenon's capsule off the conjunctiva and to shave it off the sclera completely over the area of the prospective bleb.

Operations were considered to be successful if, after a minimum period of 3 months, the intraocular pressure was less than $20 \mathrm{~mm}$.Hg (Schiötz). A partial success was a pressure of between 20 and 30 $\mathrm{mm} . \mathrm{Hg}$ while the patient was receiving miotics (gutt. phospholine iodine $0 \cdot 125$ per cent. with gutt. pilocarpine 4 per cent. twice daily) and Diamox $250 \mathrm{mg}$. three times a day. A pressure above $30 \mathrm{~mm} . \mathrm{Hg}$ was regarded as a failure. 


\section{Results}

Table II shows the results of the two procedures. Trabeculectomy alone had a success rate of 14 per cent., whereas trabeculectomy combined with drainage (filtration trabeculec- $\overline{\bar{s}}$ tomy) had a success rate of 65 per cent. Those trabeculectomy operations in which an unintentional fistula with bleb formation developed gave rise to an additional i4 per cent. of successes. The figures for the Indian group are too small to be significant and it is our $\frac{\widehat{\sigma}}{\vec{\sigma}}$ impression that these patients' eyes react to glaucoma surgery in a similar manner to those of Caucasians.

Table II Results of two types of operation

Percentages for African patients only

\begin{tabular}{|c|c|c|c|c|c|}
\hline \multirow{2}{*}{ Operation } & & \multicolumn{2}{|c|}{ Trabeculectomy } & \multicolumn{2}{|c|}{$\begin{array}{l}\text { Filtration } \\
\text { trabeculectomy }\end{array}$} \\
\hline & & African & Indian & African & Indian \\
\hline No. of operations & & 36 & 6 & 29 & 6 \\
\hline Success & $\begin{array}{l}\text { No. } \\
\text { Per cent. }\end{array}$ & $\begin{array}{r}5 \\
14\end{array}$ & $\mathbf{I}$ & $\begin{array}{l}19 \\
65\end{array}$ & 4 \\
\hline $\begin{array}{l}\text { Success with } \\
\text { unintentional blebs }\end{array}$ & $\begin{array}{l}\text { No. } \\
\text { Per cent. }\end{array}$ & $\begin{array}{r}5 \\
14\end{array}$ & $\mathbf{I}$ & - & - \\
\hline Intermediate & $\begin{array}{l}\text { No. } \\
\text { Per cent. }\end{array}$ & $\begin{array}{r}7 \\
19\end{array}$ & 2 & $\begin{array}{r}4 \\
14\end{array}$ & - \\
\hline $\begin{array}{l}\text { Intermediate with } \\
\text { unintentional blebs }\end{array}$ & $\begin{array}{l}\text { No. } \\
\text { Per cent. }\end{array}$ & $\begin{array}{l}\mathbf{I} \\
3\end{array}$ & - & - & - \\
\hline Failure & $\begin{array}{l}\text { No. } \\
\text { Per cent. }\end{array}$ & $\begin{array}{l}18 \\
50 \\
\end{array}$ & 2 & $\begin{array}{r}6 \\
2 \mathrm{I} \\
\end{array}$ & 2 \\
\hline $\begin{array}{l}\text { Failure with } \\
\text { unintentional blebs }\end{array}$ & & - & - & - & - \\
\hline
\end{tabular}

Table III shows the number of eyes in the filtration trabeculectomy operation that

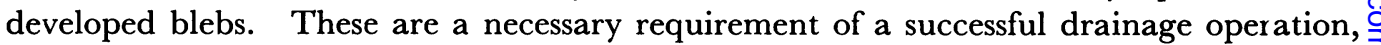
and out of nineteen successful operations there were thirteen with blebs and six without. It was pleasing to see that with filtration trabeculectomy there was such a high rate of bleb formation and that the trabeculectomy did not interfere in its formation. At the same time, failure of bleb formation still allowed six successes out of 29 eyes (20 per cent.). N It is therefore apparent that trabeculectomy in itself has a low rate of success, but that its mechanism of lowering the intraocular pressure still works when it is combined with drain-N age, since certain cases are successful even though there is no bleb formation. Therefore to combine the two operations is to obtain the best of both. Complications in the combined procedure are not noteworthy and formation of the anterior chamber was not longer delayed than it would have been in the conventional drainage operations. The immediate $\stackrel{?}{+}$ complications of the trabeculectomy operation depend on technique and include inaccurate positioning of the scleral flap; inadequate removal of trabecular tissue; damage to the $\stackrel{\mathbb{Q}}{\mathbb{Q}}$ lumen of Schlemm's canal; damage to lens or vitreous. The delayed complications may result in failure of the trabeculectomy to function and these causes are shown in Table IV. The commonest causes of failure of the trabeculectomy to function are: 
Table III Bleb formation in filtration trabeculectomy (35 operations)

\begin{tabular}{|c|c|c|c|c|}
\hline \multirow{2}{*}{$\frac{\text { Race }}{\text { Blebs }}$} & \multicolumn{2}{|c|}{ African } & \multicolumn{2}{|c|}{ Indian } \\
\hline & res & No & res & No \\
\hline Success & 13 & 6 & 3 & I \\
\hline Intermediate & 4 & - & - & - \\
\hline Failures & - & 6 & - & 2 \\
\hline
\end{tabular}

Table IV Causes of failure in 24 trabeculectomies

\begin{tabular}{lll}
\hline Obstruction to the openings of Schlemm's canal & & No. of cases \\
\cline { 1 - 1 } I. Peripheral anterior synechiae & & 4 \\
2. Ciliary processes & & 3 \\
3. Exudate & \\
4. Hyphaema & I \\
5. Vitreous & I \\
6. Lens capsule adhesion & I \\
7. Trapdoor incorrectly placed & II \\
8. Internal scleral fibrosis & Total & 24 \\
\hline
\end{tabular}

(a) Uveal tissue, either ciliary body processes or peripheral anterior synechiae that form and occlude the cut ends of Schlemm's canal despite iridectomy and immediate reformation of the anterior chamber. Failure of function develops within 6 months.

(b) Internal scleral overgrowth that replaces the excised trabecular space with fibrous tissue from its internal surface. This is very evident on gonioscopic examination. Failure of function occurs between 12 to 18 months.

\section{Discussion}

If the site of obstruction in open-angle glaucoma is at the level of the trabecular meshwork, then trabeculectomy is theoretically advisable. This is especially true when one knows that fibrosis of drainage areas occurs so readily through reaction from sclera and Tenon's capsule. Trabecular operations have other undoubted advantages over conventional drainage procedures. The anterior chamber re-forms immediately and the postoperative reaction is minimal. It was therefore disappointing to find that the operation was so unsuccessful. The findings here of a 14 per cent. success rate are in contrast to those of Cairns ( 1969 ), who found that 50 per cent. of 49 trabeculectomies were controlled without bleb formation. Since a total of 128 trabeculectomies were performed with and without fistula formation, in is unlikely that technical factors would contribute to the poor success rate of trabeculectomy in this presentation. If racial factors are present, and $I$ feel that there is more excessive scleral reaction in these patients, then it requires very little reaction to close the lumen of Schlemm's canal. That the free cut ends do close is evident, because initially blood could be demonstrated to flow from Schlemm's canal into the aqueous of the anterior chamber in some of the cases within the first few postoperative weeks with routine gonioscopy. It was not possible to demonstrate this phenomenon when these patients returned for follow up. The overgrowth of scleral tissue is very evident on the internal surface when it occurs. Initially a gap $5 \mathrm{~mm}$. square can be seen gonioscopically, running through the trabecular area to the ciliary body. This area slowly fills with scleral tissue and after 18 months it is sometimes completely filled in. In many cases this did not happen, but there is no way of establishing this behaviour pattern for each eye.

The combined operation of filtration trabeculectomy gives an alternative outflow and it should be suitable for cases that have a reputation for poor drainage, e.g. Negro patients or those with failed glaucoma operations on the opposite eye. The combined operation behaves postoperatively like a simple drainage operation although the bleb is probably flatter. 
Krasnov (1968) suggested that certain glaucomas were due to obstruction in efferen⿳亠丷厂 channels distal to Schlemm's canal in the sclera. In these cases, gonioscopy shows the trabecular area to be heavily pigmented, indicating that pigment particles can enter the trabecular area but are blocked from leaving the distal exit pathways. Sinusotomy of externalization of Schlemm's canal would then be indicated. Unfortunately, such aw operation is unlikely to succeed in African patients since a sinusotomy is certain to fibrose and close. One should also consider trabeculotomy as an operation of choice and m䞴 experience is limited in this field, but as a trabeculectomy can close from overgrowth of scleral tissue from the internal surface, so trabeculotomy with a much narrower cleft cam close. Krasnov (1969) stated that there was a marked tendency for the cleft to become $\vec{f}$ obliterated, yet Harms and Dannheim (1969) had an 85 per cent. success in cases of open-angle glaucoma. Redmond Smith ( 1969 ) found that trabeculotomy had a bette chance of lowering the intraocular pressure than conventional fistulizing surgery an's said that racial factors did not affect the prognosis. Trabeculectomy in a specific raciat group therefore appears to have only a partial success since it completely controls the intraocular pressure in 14 per cent. of cases and partially helps in 19 per cent. of cases? However, by combining trabeculectomy with a drainage procedure, one gets the benefit of both mechanisms and this has improved the success rate of the drainage operation te 65 per cent. This compares very favourably with the figures of 39 per cent. for trephines 26 per cent. for filtering iridectomies (Scheie), and 4 per cent. for iridencleisis (Berson $\vec{e}$ Zauberman, Landau, and Blumenthal, I969).

\section{Summary}

Two groups of African patients with chronic open-angle glaucoma were operated on The first group was treated by the operation of trabeculectomy, and the success rate iro cases without fistula formation was 14 per cent. A further 14 per cent. were successfu because of the formation of unintentional drainage blebs. The second group was treateक by the operation of trabeculectomy combined with intentional fistula formation (filtration trabeculectomy), and the success rate was $6_{5}$ per cent. It was concluded that trabeculectomy on its own is only partially successful, but it can be combined with a drainage pro응 cedure which will improve the results of the drainage operation in a group of patients who have a low success rate normally.

I wish to thank Prof. L. Baker, Head of the Department of Surgery, and Dr. H. Wannenburg, Superintendent of King Edward VIII Hospital, for permission to publish these cases.

\section{References}

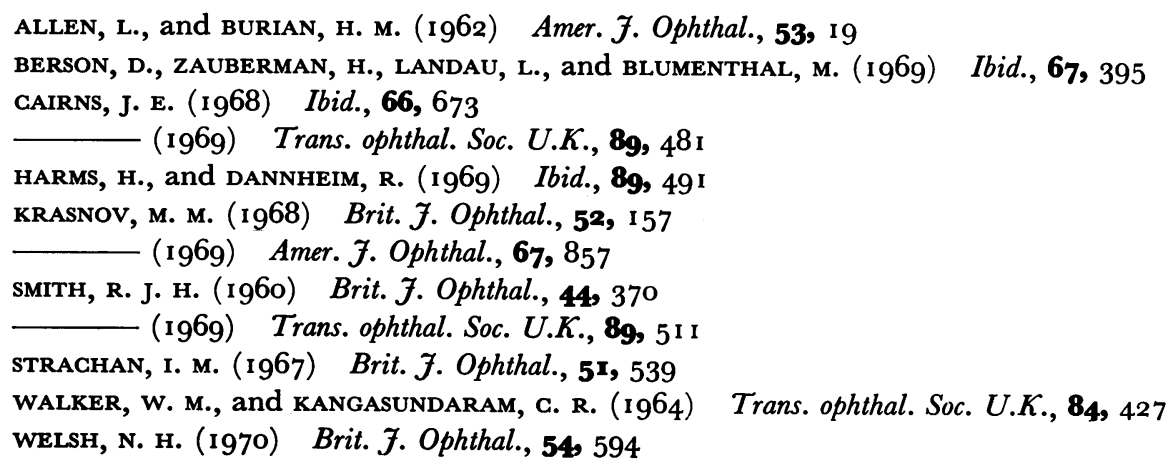

